\title{
Chapter 7 \\ Searching for New Aesthetics: Unfolding the Artistic Potential of Images Made by the Scanning Electron Microscopy
}

\author{
Anastasia Tyurina
}

\begin{abstract}
The visual arts have become a powerful tool for alternative approaches to scientific outputs, but it is crucial that both science and art cultures are aware of their interdisciplinary capabilities and limitations. It is necessary to differentiate between images captured by devices designed as resources for scientific investigation and images that exploit the 'visual elements' of scientific images. A great example of a device designed for scientific exploration is the Scanning Electron Microscope (SEM), which was introduced to scientific research in the mid-1960s. This chapter outlines the procedures, processes, and methodologies used in developing a body of studio work that investigates the artistic potential of scientific images made by the Scanning Electron Microscope (SEM) with a view to its possible social and cultural impact of this practice. It also outlines the developed theoretical findings and proposes that certain images made by the SEM can have esthetic value apart from that of scientific documentation. The use of artistic manipulations in experiments with the SEM fuses science and technology with art, and the SEM-based images that result provide a new meaning for scientific photomicrographs.
\end{abstract}

Keywords Science-art • Visual arts • Photomicrography • Interdisciplinary • Microworld $\cdot$ Interactivity $\cdot$ Aesthetics $\cdot$ Self-organizing processes $\cdot$ Coffee ring effect $\cdot$ Alternate visualizations $\cdot$ Visual coalescing $\cdot$ Creative coding $\cdot$ New media $\cdot$ Scanning electron microscope $\cdot$ Water

\subsection{Artist Statement}

My art practice involves interplay between photography and scientific imaging, photomicrography in particular. Originally being a technical discovery, photography has been widely used in almost all fields of human activity, acting as a research tool and as an independent artistic direction. Modern times offer "modern" interpretations of

\footnotetext{
A. Tyurina $(\varangle)$

Queensland University of Technology (Brisbane), Brisbane, QLD, Australia

e-mail: anastasia.tyurina@qut.edu.au
}

National Research University MIET (Moscow), Moscow, Russia

(C) The Author(s) 2020

R. Earnshaw et al. (eds.), Technology, Design and the Arts-Opportunities and Challenges, Springer Series on Cultural Computing, https://doi.org/10.1007/978-3-030-42097-0_7 
scientific photographs and attitudes toward them. Scientific photomicrography is a powerful tool for receiving and storing information and for providing solutions to a variety of tasks in many areas of science and technology. At the same time, the artistic application of photomicrography is capable of revealing a set of complex and interrelated principles that underlay the materiality of the human environment. Photomicrographs also expand human visual vocabulary, revealing principles of beauty which are difficult to access with the naked eye.

Water is the main subject of my research project. I believe that an interdisciplinary approach is the most appropriate one for deepening knowledge about unique properties of water and building a sustainable practice of water management through enhanced visualization of water contamination. My focus is on interconnected artistic thinking and by developing alternate forms of visualization, I aim to transcend disciplines and contribute to the new ways of seeing water.

My visual artworks consist of series of images made by the SEM depicting the nature of water (water chemistry), which is invisible to the naked eye, through using the SEM utilizing the phenomenon of drop evaporation. However, it was not the purpose of this research to claim that the created images of water after evaporation are scientifically valid forms of documentation; rather, this research takes an esthetic approach to scientific photomicrography.

\subsection{Introduction}

This research was undertaken during my Ph.D. study at Queensland College of Art, Griffith University and involved esthetic approaches to scientific photomicrography. Scientific photography is commonly perceived as a way of recording scientific data through techniques such as photomicrography, high-speed photography, time-lapse photography, X-ray photography, and aerial photography, among others.

Images made by the SEM are not photographs in the traditional sense; they go beyond what can be captured with light because the process of producing a picture is camera-less. Imagery produced by the SEM can confuse the viewer because the microscopic sample seems as if it is observed in the eye aperture when illuminated, and light seems to come from a particular illuminant. SEM photomicrographs are constructed out of pixels synchronized with a distribution map of the intensity of the signal being emitted from the scanned area of the specimen [1].

Interestingly, the last decades' use of SEMs in creating scientific images formed a new, well-established visual culture within a variety of scientific disciplines. As Klaus Hentschel explains, it became "an image centered science in the sense of being even totally dependent on photographic images as basis of all further processes of inference" [2, p. 315].

By exploring the interplay between the indexical and iconic modalities in scientific photomicrographs, I try to imbue them with new meanings. Both art and science are experimental in nature. There are different ways of artistically representing and perceiving an object, some of which may be valuable for science. My practice aims 
to draw attention to the qualities of water through enhanced visual details that aid in the interpretation of and differentiation between water samples.

This project investigates how to reinterpret photomicrographic images made by the SEM of micro-scale drops of water after evaporation and thus turns scientific photography into an art form.

In recent decades, there have been increasing concerns over the ecological management of water. Waterway pollution is recognized as placing urban ecosystems around the world at risk. Rainfall that washes oils, metals, and nutrients directly from streets into rivers and seas is hard to treat [3]. This is a challenging problem for science and technology. The health and well-being of present and future generations are dependent on how quickly and well it is managed.

Photomicrography has a particular potential to respond to this issue from both a scientific and a cultural perspective. SEM-made photographs are capable of visually representing features related to water composition and, in some cases, the contamination of water. At the same time, they can transmigrate from science into the domain of art and draw attention to water issues.

\subsection{Water Represented by the Scanning Electron Microscope}

The variety and frequency of the unusual properties of water are determined by the physical nature of its atoms and their association in the molecule and the groupformed molecules $[4,5]$. The composition of water, even that which is entirely free from mineral and organic impurities, is complex and diverse because water is constantly in contact with many substances.

During my experimentation with the water droplets collected from different aquifers, I aligned the scientific method of revealing water composition (the so-called 'Coffee Drop Effect') with my artistic practice. The drop evaporation phenomenon, the so-called 'Coffee Ring Effect', has been the subject of studies in the last few decades. It was first mathematically described by Robert D. Deegan in 1997 as a natural model for studying the dynamics of self-organizing processes and is actively used in physical experiments [6]. When the liquid is 'pinned' to its contact surface, the liquid evaporating at the exterior edge is replenished by the constant flow of liquid from the interior to the periphery. This flow carries nearly all dissolved solids toward the edge [7].

During experiments for my project, the structure of the water impurities visually transformed, leading to a unique connection between the processes of evaporation and solidification. These two processes are shown in Fig. 7.1. This natural process of drying reveals the unique informative capacity of droplets through the shapes, patterns, details, and characteristics resulting from each water sample.

At the University of Chicago Materials Research Center, scientists are exploring the driving mechanism responsible for this phenomenon that is found in many 


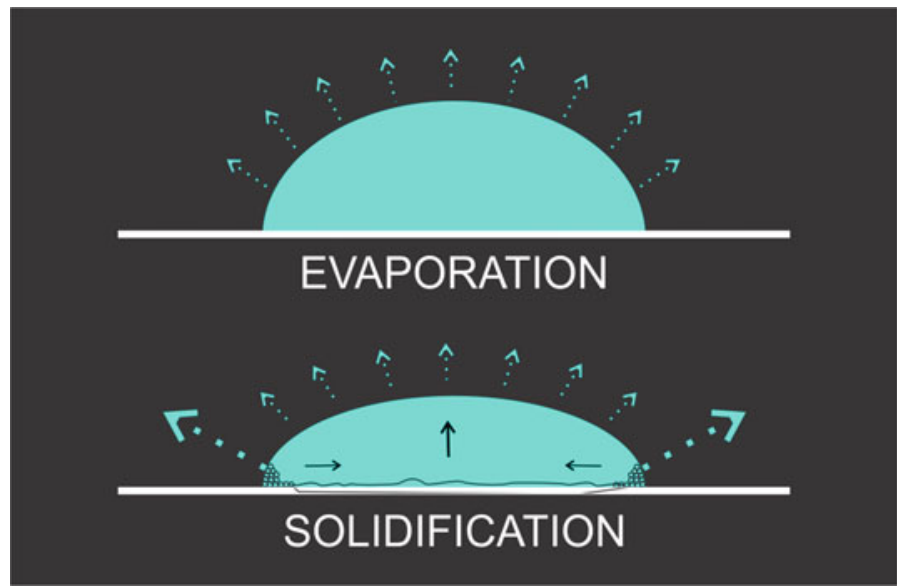

Fig. 7.1 Anastasia Tyurina, Drop Evaporation and Solidification Scheme, 2015. Image copyright (C) A. Tyurina 2019

varieties of liquids. This research validated my process of exploring variations in water composition for my project. On its webpage, the Center states: "We have determined that ring formation is a ubiquitous and robust phenomenon. It doesn't depend on the solute, the solvent, or the substrate so long as the solvent is partially wetting and volatile, and the contact line is pinned" [8].

An interesting observation made by the researchers is that "By controlling the speed and spatial variation of the evaporation, this model predicts that we can control the shape and thickness of the deposit" [7]. This was particularly important for my practice because it meant that experimenting with various modes of evaporation could lead to different visual formations of the drops after they dry.

Specifically, for this research 'science' can be described as a process through which new knowledge about the world is built. The scientific process relies on the testing of ideas through experimentation. Scientific photography, as a tool, aims to support the scientific process by capturing data about the world for examination.

Reflecting on the scientific findings, the primary purpose of my visual art project was to depict the inherent features of water that are invisible to the eye through using the SEM. To do so, I used the process of evaporation as an alternative and unusual artistic method of visually presenting the composition of water. My approach is unique in the specific way in which I use water to create images using the SEM. During experiments for my project, it became evident that the structure of water impurities is visually transformed after evaporation and reveals a unique connection between evaporation and solidification. This process of revealing the nature of water (water chemistry) allowed me to play with the process like an artist [9].

One of the technical requirements when taking a microscopic image with the SEM is that any object placed in the chamber of the SEM must be completely dry, because the SEM operates in a vacuum. As explained in Under the Microscope: A 
Hidden World Revealed (1987), "Turning to biological specimens for the SEM, it used to be necessary first to fix and dehydrate them, and then dry them either on air or by the use of liquid carbon dioxide ('critical point drying')" [10, p. 199]. After the evaporation process, water is no longer a liquid; dry solids or other substances become watermarks that represent its previous composition.

The main difference between 'Artistic' and 'Scientific' use of the SEM in this project is that the artistic approach has a focus on making aesthetic images. Within this is the substance of the scientific idea about showing water state and how it is changing. In my work the results of imagery processes become art pieces with both artistic and scientific applications (Fig. 7.2).

My artistic intervention into a scientific process through experimenting with the SEM was a way to find out what different things my images could reveal about water to a viewer. There was an opportunity to explore some concerns related to the environmental impact of water pollution in an artistic context and to communicate the significance of water.

By exploring the integration of art and science in this way, my project focuses on the use of scientific tools to create science-based art. However, it must be recognized that my approach was primarily one of artistic research and experimentation: a search for new aesthetics, exploring spatial and temporal dimensions, engaging with materiality, and involvement of modern technologies in the formation of esthetic experiences for the viewer. In my project, images are highly variable in what they show and how they show it because of changes in water content.

\subsection{Photomicrography as an Art Form}

Contemporary practices in photomicrography are making significant contributions to the dialog around aesthetics and artistic components in science. As far back as 1963, in his article "The Esthetic and Pictorial Applications of Photomicrography" for The Photographic Journal, Douglas Lawson wrote:

When mentioned within the hearing of some pictorialists they immediately think it is only for the scientifically minded, or the record worker. Nevertheless, the so-called record photograph can be made to look quite attractive. Some of you may remember Mr. H. A. Murch, one of our great pictorial photographers, once saying, 'I do understand the desire to apply pictorial ideas in record work, which is a very different objective, and we ought to welcome such an application when it can be done without losing anything of the essential factual value of the record' Photomicrography offers expression not in what the painter has already done but in what the painter cannot do. Because this application of photography is highly scientific there is perhaps a tendency to think of it as being without scope for the artistic application. May I suggest that a work of art in any medium can be the deliberate creation of unity, and nature through the microscope is one medium provides us with plenty of scope for such unity [11].

To effectively engage with photomicrography as a social phenomenon, it is crucial for an artist to demonstrate an understanding of its 'scientific' protocols of representing. But will the viewer be familiar with what he or she sees in scientific images? If 


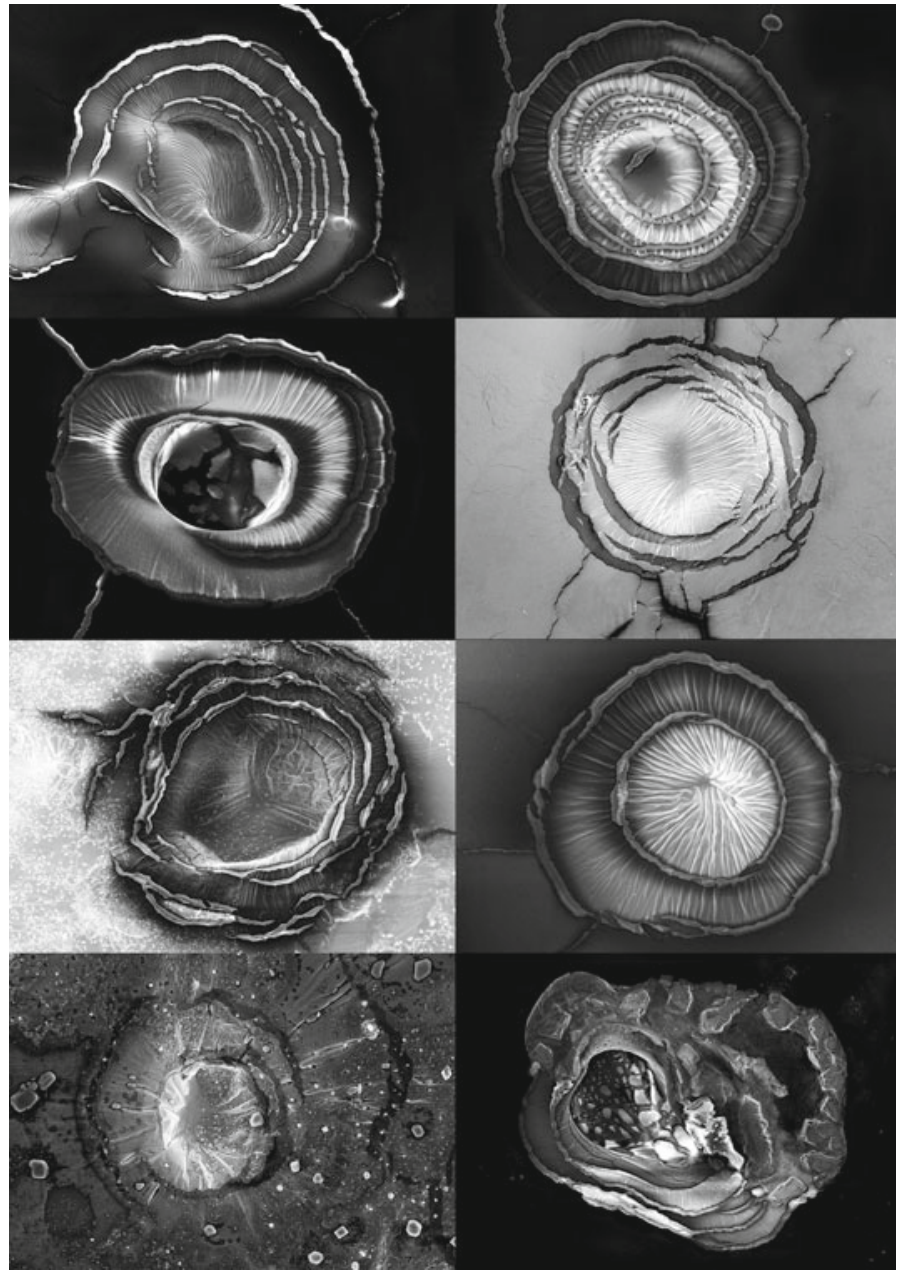

Fig. 7.2 Anastasia Tyurina, Watermarks Series, 2015 (From left to right: Brown Lake, Brisbane River, Raby Bay, Rainwater, Mount Gravatt Pond, Mimosa Creek, South Bank Pond, Cylinder Beach). Image copyright $\odot$ A. Tyurina 2019

not, how will they perceive such images? Do the images need to be explained or are they capable of conveying messages in a different context?

Artistic photomicrographs of water after evaporation call upon the viewer's creative ability to perceive previously unseen water composition after evaporation as well as to observe natural phenomena over and beyond the directly visible.

My images transform the microworld to a macro-level and evoke an interest in water chemistry that is shown as being beautiful. This causes a dilemma for viewers, particularly because the gallery space is different from a laboratory. The captions for my photographs refer to the sites where the water samples were collected. It 
is intriguing that they can resemble aerial photographs of topographical features of particular water reservoirs (Figs. 7.3 and 7.4).

Today, the practice of visually pleasing photomicrography is more mainstream. The most popular international photomicrography competition is Nikon's Small

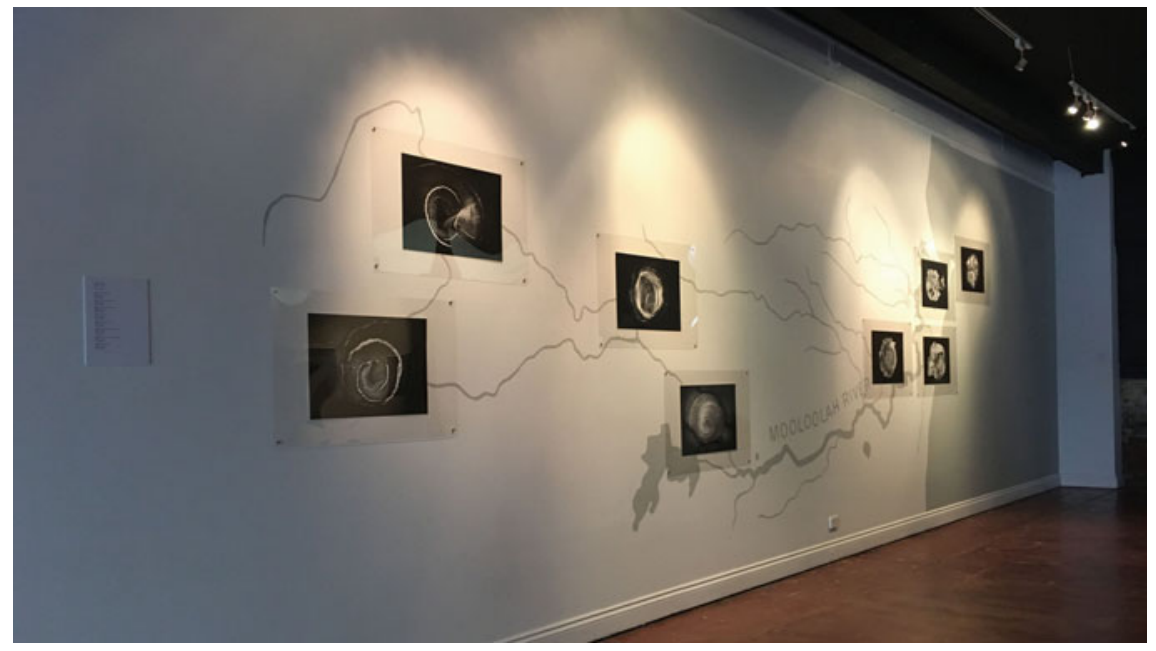

Fig. 7.3 Anastasia Tyurina, Photo documentation of the artistic installation for $\mathrm{H} 2 \mathrm{O}+$ exhibition, PoP Gallery, Queensland College of Art, Griffith University, 30 March-16 April 2017. Image copyright () A. Tyurina 2019

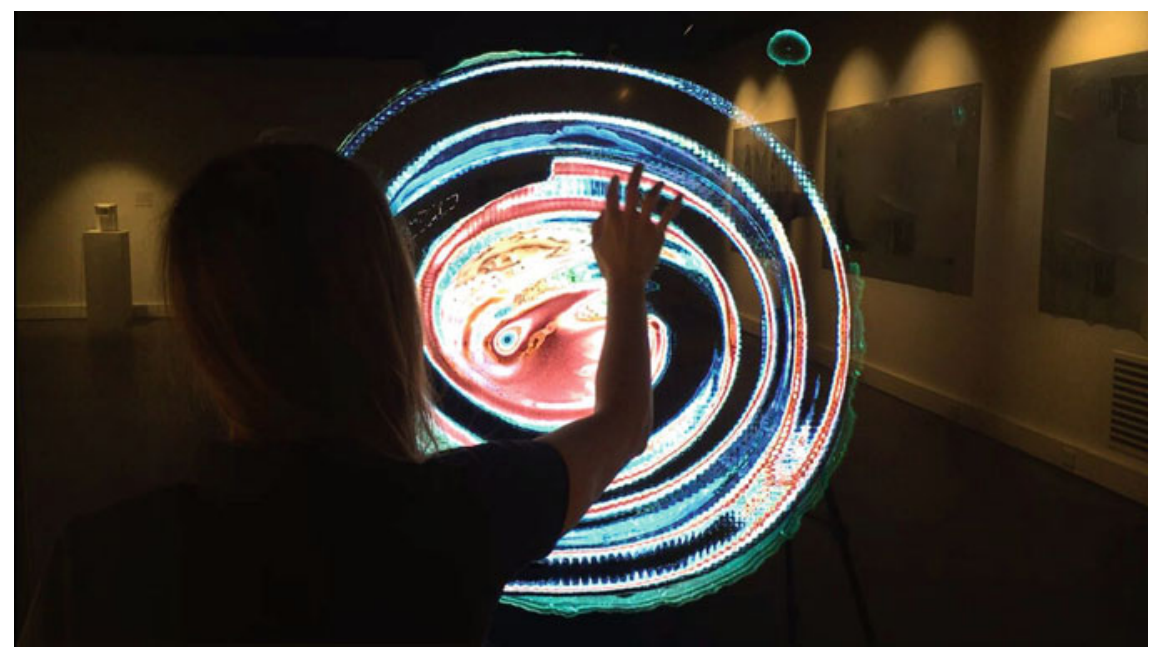

Fig. 7.4 Anastasia Tyurina, Photo documentation of the Shifting the Posts exhibition, Webb Gallery, Queensland College of Art, Griffith University, 27 November-7 December December 2018. Image copyright (C) A. Tyurina 2019 
World Photomicrography Competition, which dates back to 1975. Participating images are judged for "their scientific and artistic merits" [12] and represent a broad range of visual studies of the microworld. Some of these photomicrographs have allegorical titles, a fact that highlights the relation of artistic intentionality in producing images and the scientific objectivity underlying the concept of the competition.

Participation in the competition is restricted to images made by optical microscopes of any kind, which are capable of revealing natural colors of objects. As mentioned above, the SEM apparatus is camera-less; there is no light involved in the process; it is not an optical instrument. Thus, colors cannot be reproduced. Rather, artificial colors can only be added with the help of graphic software such as Adobe Photoshop. While there is a whole range of possible manipulations available in Photoshop, it is worth mentioning the coloring tools. Through different algorithms, it is possible to apply different colors to different details of the image. There are many sources both in the technical literature and on the Internet providing scientists and artist with various tutorials on image coloring. But the author of the image makes the final decision of which color to apply to a particular point of the presented object and thus color choice is very individual. Experimenting with such coloring, I have tested two options: the coloring algorithm available in Photoshop and the software offered by Recolored, which was designed for the purpose of recoloring black-and-white photographs (Fig. 7.5).

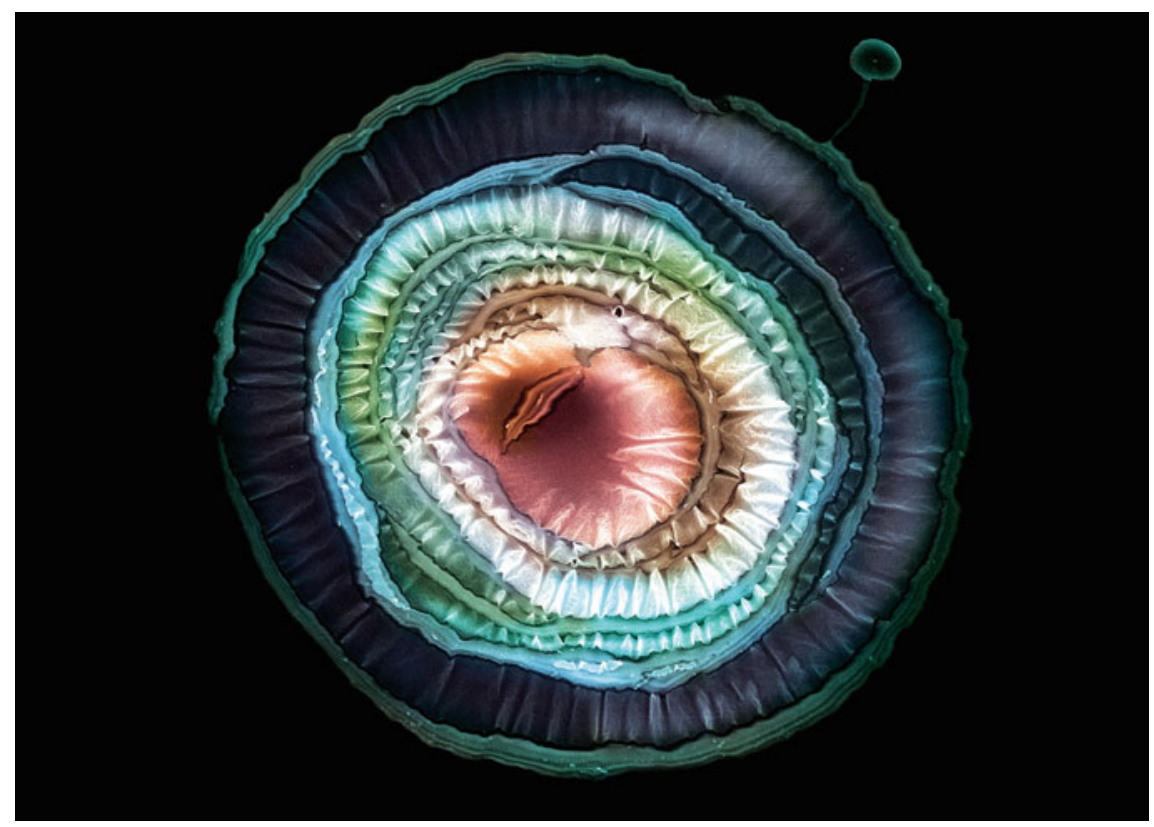

Fig. 7.5 Anastasia Tyurina, Colour 32, 2017. Image copyright ( A. Tyurina 2019 


\subsection{Photographs or not Photographs?}

It is important to examine the process of image formation in relation to the imagery obtained by the SEM in modern microscopy. It is also crucial to distinguish image generation by SEM considering that the process is not one that is based in code. The image generation process can be seen as a work of high-energy electrons that create a range of different signals when hit the specimen. Converted into pixels signals result in image formation appeared on the screen.

Technically, the SEM produces electron micrographs because the image is developed by either secondary electrons (electrons ejected from the material by the incoming electron beam) or backscattered electrons (electrons from the electron beam, which have 'bounced' off the material into the detector). Dee Breger explains:

Scanning electron microscopes don't merely use electron beams to illuminate objects so small they can't be seen by light. Since they are electronic devices, SEMs can manipulate isolated groups of electrons from the sample-beam interaction to create separate pictures (such as secondary and backscatter images) that contain different kinds of information about a single object. This variety can lead to a 'compound reality' or, since no version tells the whole story, a kind of 'ironic nonreality' [13, p. 11].

Images produced by the SEM are beyond light; captured by a focused beam of electrons, they are not photographs. The apparatus tries to recreate a reality that is not a visual phenomenon, which scientists then try to analyze through its visual representation: the photomicrograph.

However, the most commonly used SEM electron detector, named EverhartThornley (E-T), ${ }^{1}$ typically uses a material that produces light when an electron collides with it-a scintillator. ${ }^{2}$

Therefore, the SEM does use photons, but they are converted back into electrons, which are accelerated onto the electrodes of the photomultiplier, producing an increasing stream of electrons until the final collector is reached [14]. The image formation in scanning electron microscopy consists of the scanning system, the signal detectors, the amplifiers, and the display [1]. A physical, material connection of the SEM apparatus with the studied object is gained by the interaction of these four elements (Fig. 7.6).

\footnotetext{
1 "The E-T detector operates in the following manner: when an energetic electron $(\approx 10 \mathrm{keV}$ energy) strikes the scintillator material (S), light is emitted. Light is conducted by total internal reflection in a light guide (LG) (a solid plastic plastic or glass rod) to the photocatode of a photomultiplier (PM). At the photocatode, the photons are converted back into electrons, which are accelerated onto the successive electrodes of the photomultiplier, producing an ever-increasing cascade of electrons until the final collector is reached" [14].

2"A scintillator is a material that accepts incident high-energy electromagnetic or charged particle radiation and in turn uses that energy to fluoresce photons whose peak emission wavelength is longer than the wavelength of the incoming radiation. In the case of a SEM, the scintillator disc collects the secondary electrons that are produced as the electron beam scans the surface of the sample. These electrons are converted into photons which travel through the light pipe to the photomulitiplier tube (PMT) so that the signal may be amplified to the level required for viewing" [15].
} 


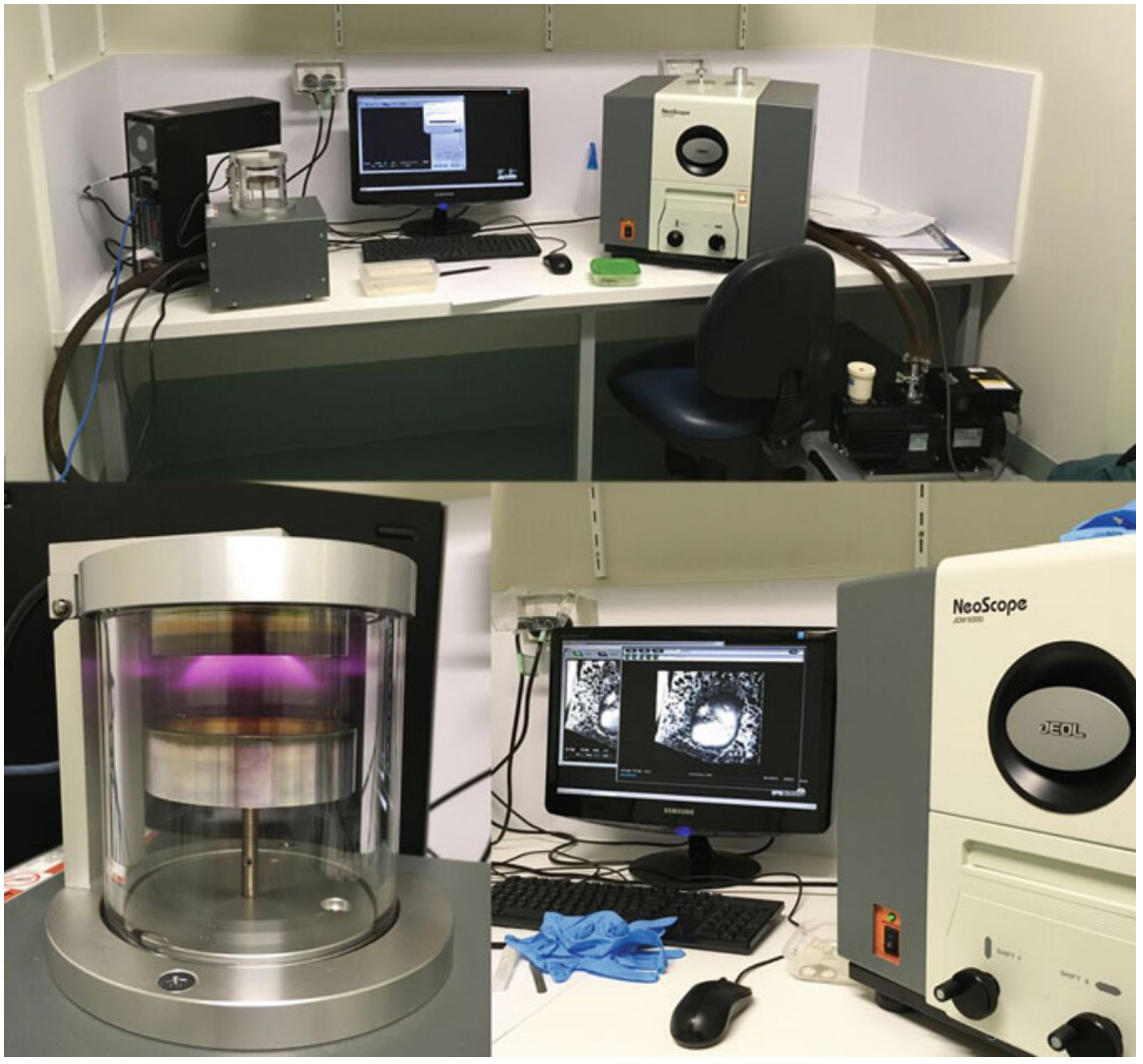

Fig. 7.6 Anastasia Tyurina, Scanning Electron Microscope Imaging Facility, 2016. Image copyright $\odot$ A. Tyurina 2019

During the imaging process, the energy exchange between the electron beam and the sample results in the reflection of high-energy electrons, the emission of secondary electrons and the emission of electromagnetic radiation, each of which can be measured by specialized detectors. The detectors interpret these signals by the algorithm and the resultant image appears constructed out of pixels. This algorithm is defined by each quantum of signal information but it is also implemented in the physical world in apparatus, computer, and screen [1]. In some sense, the SEM apparatus interprets (transforms) signals and visualizes signals.

\subsection{Scientific Images and New Media}

The artworks employ two kinds of medium, comprising two different applications of SEM photomicrography: still photomicrographs and interactive digital installations. They can be perceived differently in terms of the esthetic response they generate in 
the viewer, but both draw attention to the qualities of water through enhanced visual details that aid in the interpretation of water samples. I use static SEM-generated photomicrographs of water, then apply a digital code which allows the image to be altered by the audience's interaction with its touch-sensitive interface. Thus, anyone who touches the screen can create, display, and experience a ripple effect, which is very similar to the effect we can see and observe when we interact with water surface by touching or disturbing it. Interacting with the scientific photomicrographs in this way offers a layered meaning and can enhance the audience's perception of scientific data, scientific photography, and water.

Exploring the idea of 'interactivity' for my project, I looked for a method that allows viewers to interact with photographs by altering the work's visual content. Utilizing the programming language Processing, I developed an algorithm which allows viewers to physically interact with my photomicrographs so that they become direct objects of manipulation. Using the random human touch of some areas of the developed algorithm alters the static image, which in some senses visually represents non-visual elements: scientific data.

Through my research, I have explored different digital tools and realized that creative coding suits my goals because this type of computer programming is designed to create something expressive instead of something functional. Using creative coding tool such as Processing, it is possible to create art installations, projections, sound art installations, and much more within the context of the visual arts.

The interplay between virtual ripple effects and the resemblance of physical interaction with water can be seen as embodying the "artist-display-user" paradigm seen with regard to computer art [16, p. 10]. In their article "The Post-Display Condition of Contemporary Computer Art," Toby Juliff and Travis Cox attempt to re-conceptualize the relationship between artistic intentionality, coding, interface, and user input. They suggest that the relationship between the artist and computer code should be reconsidered so that the paradigm becomes "artist-code-display-user." They state that "the code takes input from the user and subjects it to internal semiosis between distinct elements of the code, before being output to the display. This code, having been written by the artist, or an agent of the artist, contains within its structure an inbuilt intentionality, a way of approaching input that is integral to conveying the intended meaning" [16, p. 10]. In line with artists using code to activate new experiences and with Juliff and Cox, who argue that the code is not only a product of intention and meaning but also a producer of them, I seek in my practice to explore the role of code in the production of meaning, in particular for the scientific photomicrograph of natural phenomena.

In the series of live images for my project, the photomicrographs displayed on the screen seem to be still when viewed from a distance; yet, as the audience comes closer and start touching the screen, the ripples appear.

The photomicrograph transforms into a live picture, producing varying forms of ripple that seem both ordered and random at the same time. Such transformations occur continuously until the user-viewer stops touching the screen (Figs. 7.7 and 7.8). 


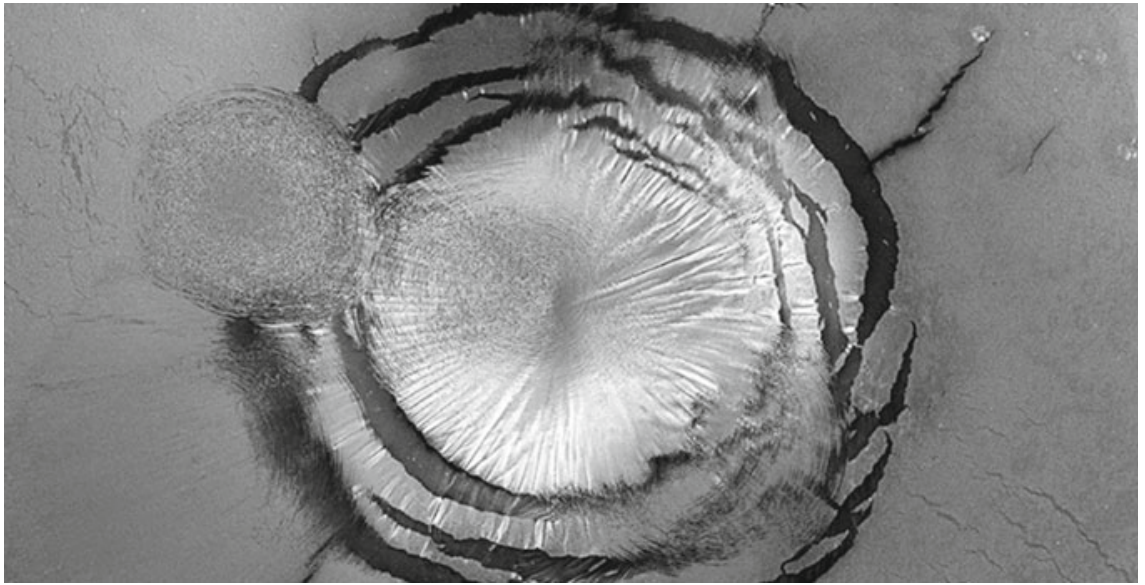

Fig. 7.7 Anastasia Tyurina, Rainwater (detail) 2016, Digital mixed media. Image copyright $(\subset)$ A. Tyurina 2019

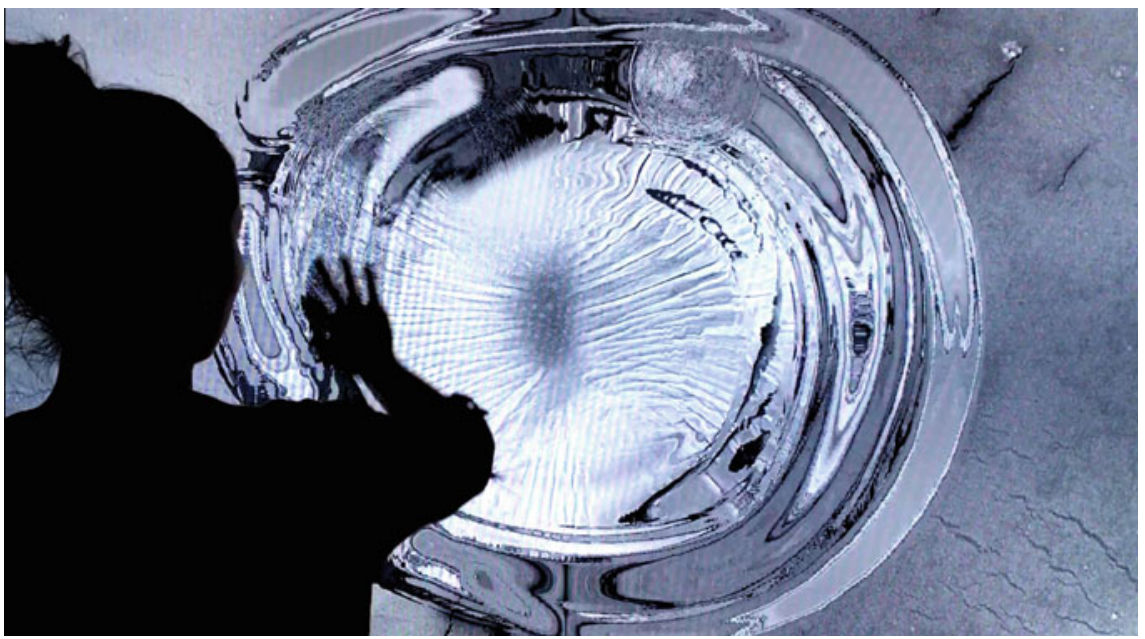

Fig. 7.8 Anastasia Tyurina, Rainwater (detail) 2016, Digital mixed media. Image copyright $\odot$ A. Tyurina 2019

Interacting with the image in this way transforms the work into something that transcends disciplines. It offers a layered meaning, providing audiences with the opportunity of experiencing the fluid and animated qualities of the effects that connects with these qualities in the subject matter: water. The addition of the interactivity and animation introduced by the ripple effect is the expanded mode of 'reading' or appreciation. As well as offering a visual engagement, the work offers an embodied 


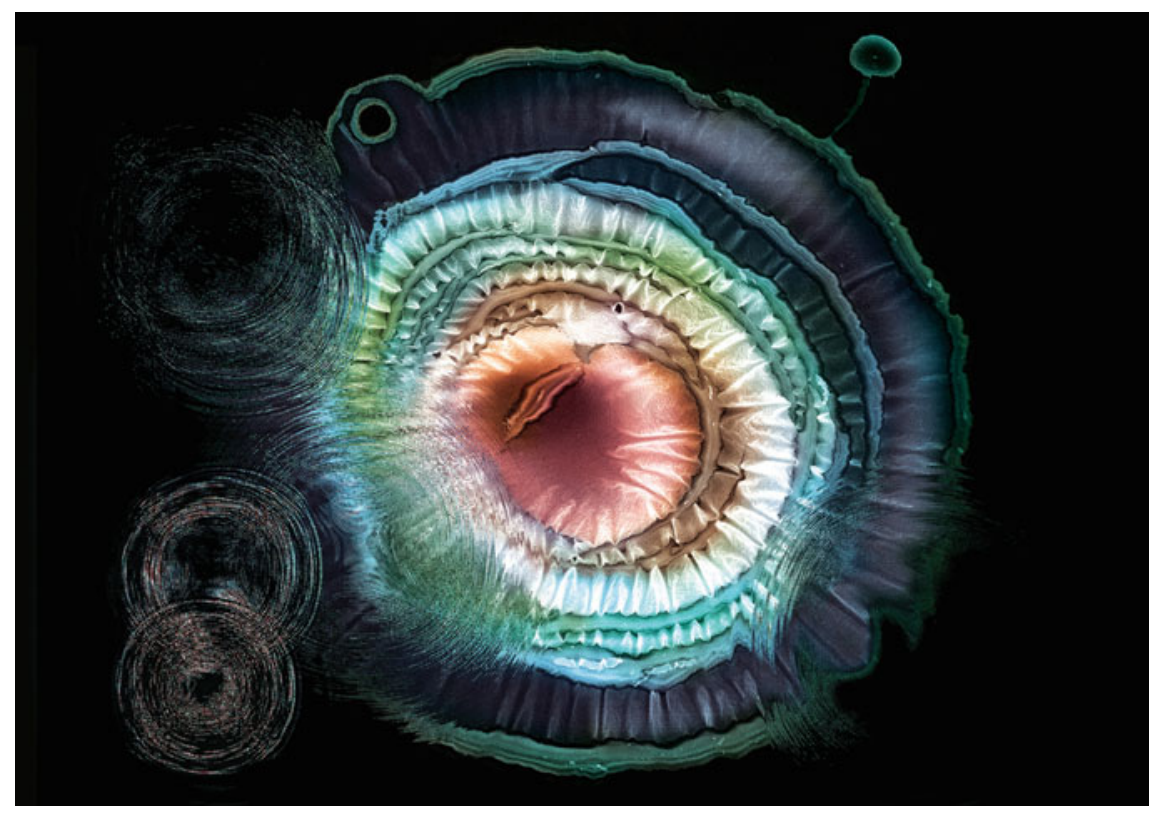

Fig. 7.9 Anastasia Tyurina, Colour 32 (detail) 2017, Digital mixed media. Image copyright $\odot$ A. Tyurina 2019

engagement: an important connection to the material significance of water in our lives.

Colour 32, one of the artworks, portrays a drop of Brisbane River water. This artwork was exhibited at various venues displayed as both interactive work and video at screens of different scale (Figs. 7.9, 7.10 and 7.11).

Such digital installations allow the transformative power of water to be explored; they offer new perceptual experiences and, by the artist-code-display-audience interaction, are capable of providing a new meaning for scientific images through their ever-changing visualization.

\subsection{Conclusions}

Not every image is art, and art is far more than just an image. Originally a technical discovery, photography has been widely used in almost all fields of human activities, acting as a research tool and as a form of artistic practice. Scientific tools have brought new ways of seeing the world. Although usually reserved for scientific use, such technology is now being used in the creation of art.

The primary purpose of this research was to show that the artistic use of SEMmade photomicrography can shift the visual outcomes of scientific photomicrography 


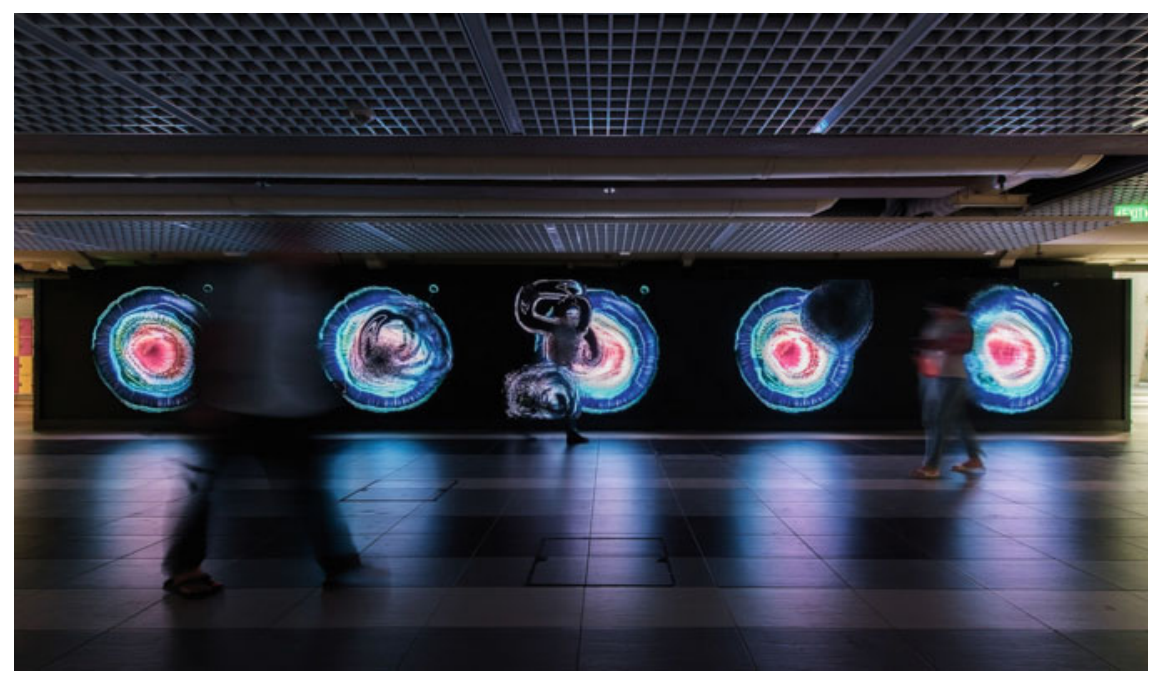

Fig. 7.10 Anastasia Tyurina, Colour 32, North Spine Plaza, Media Art Nexus, Nanyang Technological University (NTU), Singapore, 2017. Image copyright @ A. Tyurina 2019

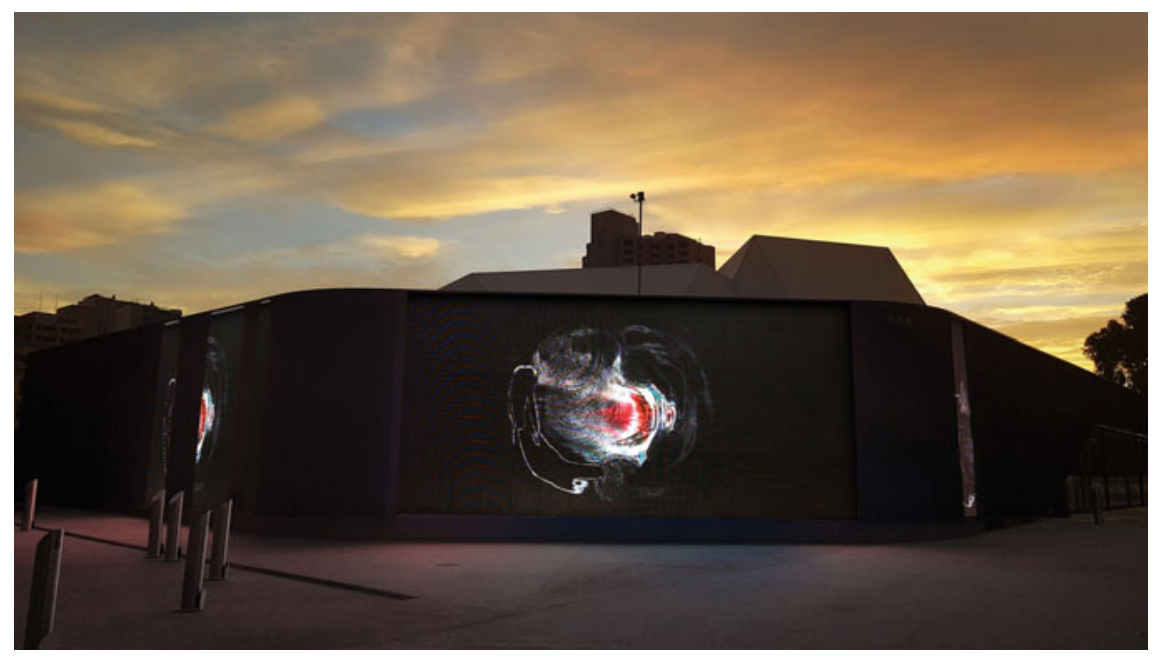

Fig. 7.11 Anastasia Tyurina, Colour 32, public screening, Adelaide Festival Centre, 2018. Image copyright $($ C A. Tyurina 2019

to function within the context of art. Drawing on literature, visual analysis, and theoretical findings, it was possible to develop my artistic practice using the SEM to visualize the microworld of water. As Breger states, the microworld exists beyond our cognition; its beauty is hidden but it can be explored using appropriate tools, such as the SEM [13]. 
Not many people have access to complex research technologies that can widen their daily experience such as the SEM. Mostly, people passively receive scientists' dry results and the interpretations they are exposed to. My work in the field of photomicrography aims to expand human visual vocabulary, revealing principles of beauty which are typically difficult to otherwise access. Thus, my artistic intention is to expand the visual representation of the composition of water, its chemical features, and their patterns after evaporation. My aims are to also communicate issues related to the ecological management of water and to raise and deepen responsible attitudes toward consuming and managing water resources.

During this project, I have placed (both virtually and physically) scientific images into a 'hostile' artistic environment. In the gallery space, the significantly greater scale of photomicrographs, their impeccable details, the artistic materials used, and the digital, still and interactive modes of presentation uncover the novel artistic potential of the scientific image. This leads to the image being disconnected from pure data and objectivity and allowing the viewer to perceive it as art, helping them to interpret photomicrographs of water in different ways. Most importantly, through these images, I hope to uncover the horizon of meanings previously unseen.

Today, many institutions and organizations host scientific photography and visualization competitions (and associated exhibitions), which encourage scientists from different disciplines to contribute. The images are not only judged by professionals (scientists and photographers) but often the audience is also invited to evaluate the competing images. By doing so, all three groups apply their own esthetic judgment criteria. This illustrates how scientific images can be evaluated from different contexts. Examples of such events include the annual the Visualization Challenge, National Science Foundation; the Wellcome Image Awards, Wellcome Trust; Nikon Small World Photo Competition; The Royal Photographic Society International Images for Science, just to name a few.

This popular trend indicates the growing interest in the visual coalescing of science and art through photography, particularly through photomicrography. Nevertheless, the idea that the photographic scientific image can be artistic and that the arts can serve science and vice versa still generates skepticism, and the debate will surely continue. Regardless, it is obvious from the discussion that the arts have the potential to reveal important aspects of the complex world in their own way, which is complementary to what scientists do and may be useful for both disciplines. Scientific photography aims to record and illustrate data and experiments that differ according to specific disciplines. Although the main purpose of scientific photography is to convey accurate information rather than beauty, its ability to record material in addition to that which is merely informative allows it also to serve expressive, subjective, and esthetic purposes.

The new definition other than photographs (or photomicrographs) for SEM images should be sought considering the nature of the process of image generation by the SEM. This is particularly interesting due to the complex nature of the apparatus. Different ways in which images made by SEM can be interpreted create layered meaning in them especially if they are given in the artistic context. 


\section{References}

1. Goldstein, J.I., Newbury, D.E., Echlin, P., Joy, D.C., Fiori, C., Lifshin, E.: Electron Microscopy and X-Ray Microanalysis: A Text for Biologist, Materials Scientist, and Geologists. Springer Science + Business Media, New York (1981)

2. Hentschel, K.: Visual Cultures in Science and Technology. A Comparative History. Oxford University Press, New York (2014)

3. De Haan, F., Rogers, B., Frantzeskaki, N., Brown, R.: Transitions through a Lens of Urban Water. Environ. Innov. Societal Transitions 15, 1-10 (2015)

4. Chaplin, M.: Water Molecule Structure. Water Structure and Science (blog). Last updated 30 October. http://www1.1sbu.ac.uk/water/water_molecule.html (2016). Last accessed 19 Jan 2017

5. Chemical Association: Accessed on 6 June 2019. https://www.britannica.com/science/ chemical-association (2019)

6. Yakhno, T.A. Yakhno, V.G., Sanin, A.G., Sanina, O.A., Pelyushenko, A.S., Egorova, N.A., Terentiev, I.G., Smetanina, S.V., Korochkina, O.V., Yashukova, E.V.: The informative-capacity phenomenon of drying drops. IEEE Eng. Med. Biol. Mag. 24(2), 96-104 (2005). https://doi. org/10.1109/memb.2005.1411354

7. Deegan, R.D., Bakajin, O., Dupont, T.F., Huber, G., Nagel, S.R., Witten, T.A.: Capillary flow as the cause of ring stains from dried liquid drops. Nature 389, 827-829 (1997)

8. The Coffee Ring Effect Accessed on: June 6, (2019). Available: https://mrsec.uchicago.edu/ research/highlights/coffee-ring-effect (2019)

9. Tyurina, A.: The unseen water: experimentation with scientific photomicrography and creative coding. In: Proc Electronic Visualisation and the Arts (EVA 2017). Available http://dx.doi.org/ 10.14236/ewic/EVA2017.15

10. Burgess, J., Marten, M., Taylor, R., McNamee, M., Stepney, R.: Under the Microscope. A Hidden World Revealed. CUP Archive, New York (1987)

11. Lawson, D.F.: The aesthetic and pictorial applications of photomicrography. Photogr. J. Off. Publ. Roy. Photogr. Soc. G B 103(1), 1-11 (1963)

12. Thompson, N.: Tiny Photographs from the 'Limbo Between Art and Science', CNN News, 30 October (2013). Available: https://edition.cnn.com/2013/10/30/world/nikon-small-worldphoto-contest/index.html (2013). Accessed on 6 June 2019

13. Breger, D.: Journeys in Microspace: The Art of the Scanning Electron Microscope. Columbia University Press, New York (1995)

14. Electron Detectors. Available: https://www.scienzemfn.unisalento.it/c/document_library/get_ file?uuid=639f02da-34b4-43b5-b595-13978bae6164\&groupId=10122 (2015). Accessed on 31 May 2019

15. Scintillators. Available: https://semicro.org/collections/scintillators. Accessed on 6 June 2019

16. Juliff, T., Cox, T.: The post-display condition of contemporary computer art. EMAJ 8 (2015) 
Open Access This chapter is licensed under the terms of the Creative Commons Attribution 4.0 International License (http://creativecommons.org/licenses/by/4.0/), which permits use, sharing, adaptation, distribution and reproduction in any medium or format, as long as you give appropriate credit to the original author(s) and the source, provide a link to the Creative Commons license and indicate if changes were made.

The images or other third party material in this chapter are included in the chapter's Creative Commons license, unless indicated otherwise in a credit line to the material. If material is not included in the chapter's Creative Commons license and your intended use is not permitted by statutory regulation or exceeds the permitted use, you will need to obtain permission directly from the copyright holder.

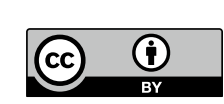

